\title{
A NUMERICAL SOLUTION TO A FREE SURFACE WAVE PROBLEM
}

\author{
A. Pawell - R. B. Guenther \\ We dedicate this work to Professor Nirenberg
}

\section{Introduction}

The aim of this paper is to present a numerical method for the calculation of water waves in a semi-infinite wave tank. The motion of the fluid, which is assumed to be inviscid, is caused by the motion of part of the boundary of the basin. The correct numerical simulation of the interaction between the side walls and the fluid is an intrinsic feature of the problem.

In the literature a series of theoretical results concerning free surface waves are presented. Most of these results deal with the fluid motion in unbounded domains or in domains bounded completely by a free surface. In these cases the boundary of the domain of fluid motion is smooth and solutions in classes of high regularity can be found. Classical existence results for a free surface problem for a viscous fluid were obtained by Solonnikov for a finite drop [19] and by Beale for an infinite domain [1]. Both authors use a mixed Euler-Lagrange formulation of the problem to transform it to a fixed domain. They apply fixed point principles to derive existence results using the smoothing effect of the friction term in the Navier-Stokes' equations and a priori estimates for related linearized problems. Under certain compatibility and regularity assumptions for the initial condition and external forces, Solonnikov proved the existence of a

1991 Mathematics Subject Classification. 35R35, 76C99, 76D33.

This work was partially supported by a Feodor Lynen grant from the Alexander von Humboldt Foundation and a grant from ONR 302623163. 
solution in Hölder spaces with $v \in C^{2+\alpha, 1+\alpha / 2}(\Omega \times[0, T])[19]$ and Beale obtained the existence of a generalized solution in Sobolev spaces with $v \in K^{r}(\Omega \times[0, T])=$ $H^{r, r / 2}(\Omega \times[0, T]), 3<r<7 / 2([1],[2])$. Here, $v$ is the velocity vector in Lagrange variables and $\Omega$ refers to the domain initially occupied by the fluid.

For the inviscid problem even higher regularity assumptions are necessary to ensure the solvability of the problem. Shinbrot obtained an existence result in the class of analytic functions for the initial value problem in a sufficiently small time interval with the aid of a power series expansion for the solution in Lagrange variables ([15], [12], [13]).

An existence result for the system of Euler equations under weaker regularity assumptions was obtained by Tani [20]. This approach is based on the classical transformation of the problem in mixed Euler-Lagrange variables used in [1], [2], [19]. The application of fixed point principles fails in the inviscid case due to the regularity defect in the resulting operator. Tani [20] provided a priori estimates for the corresponding linearized auxiliary problems under weaker regularity assumptions and used a limit analysis to prove an existence result for the free surface problem for the Euler equations with $v \in C\left([0, T] ; C^{2+\alpha}(\Omega)\right), T>0$, $0<\alpha<1$.

The reduction of these problems to a bounded domain is not just a formal problem. In any domain bounded by rigid walls, sharp corners and edges are formed at the interface between the free surface and the fixed boundaries. Natural boundary and compatibility conditions usually do not ensure such a high regularity as in the above existence results near these interfaces. For more details concerning the regularity of a solution to a partial differential equation in nonsmooth domains we refer to [4]-[6], [9].

One can deal with the loss of regularity near the edges of the domain by using weighted Hölder or Sobolev spaces. This requires information about the singularities of the solution which depend on the contact angle between different parts of the boundary. A series of existence results for the stationary NavierStokes' equations in two dimensions was obtained by Socolowsky [16]-[18].

In the present paper we derive a mathematical description for free surface waves of an inviscid fluid in a bounded or semi-infinite domain. Intrinsic features of the problem are the nonlinearity of the equations of motion and the free surface condition, the interaction between the fluid and a moving boundary of the domain as well as the hyperbolic type of the equations of motion for an inviscid fluid. These features require a special treatment and the implementation of efficient numerical methods. We derive a formulation of the problem as a system of nonlocal partial differential equation of first order for the free surface and the velocity potential. We use a transformation of the problem which splits the operator into a linear part defined in the whole domain and a nonlinear 
part in a reduced space. This transformation and the solution of the resulting problem do not require any higher regularity of the solution than that required for the formulation of the problem via partial differential equations. Furthermore, it enables us to solve the problem in a compact way. Using highly efficient implicit methods for the solution of a corresponding semi-discretized system of ordinary differential equations, we are able to solve the problem ensuring natural compatibility conditions at the interface between the fluid and the boundaries.

We solve the problem either in a bounded domain with reflecting boundary conditions or in a semi-infinite domain. The numerical solution of the second problem requires the formulation of absorbing boundary conditions at an artificial boundary simulating an undisturbed outflow condition. For this purpose we use a formal extension of the calculation domain with boundary conditions which outside the domain of interest satisfy a simple transport problem. So our method can be augmented in a natural way with an absorbing boundary condition at the artificial boundary.

\section{Mathematical model}

We consider free surface waves of water in a rectangular basin which are caused by a moving wall. For an incompressible and inviscid fluid the motion is governed by the Euler equations in a time dependent domain $\Omega(\eta, a)$. The domain of fluid motion is bounded by a fixed boundary $\Gamma_{0}$, consisting of a rigid bottom $\{Y=-h\}$ and two side walls, $\left\{X_{2}=0 \vee X_{2}=b\right\}$, by a moving boundary $\Gamma_{w}:\left\{X_{1}=a(t)\right\}$, and by a free surface $\Gamma_{s}:\{Y=\eta(X, t)\}$. We consider either an open domain with $X_{1} \rightarrow \infty$ or a closed domain with the boundary $\Gamma_{1}: Y=\gamma\left(X_{1}\right)$ opposite to the wavemaker (see Figs. 1, 2). We suppose that $g\left(X_{1}\right)$ is constant in the three-dimensional case and nondecreasing in the twodimensional case.

Under the assumption that the motion is irrotational, the equations of motion can be reduced to the Bernoulli equation for the velocity potential $\varphi$, which is coupled with a continuity equation:

(1) $\varphi_{t}+\frac{1}{2}|\nabla \varphi|^{2}+\frac{p}{\varrho}+g Y=0, \quad \Delta \varphi=0, \quad$ in $\Omega(\eta, a), \quad$ with $v=\nabla \varphi$,

where $v$ denotes the velocity vector, $\varphi$ the velocity potential, $p$ the pressure and $g$ the acceleration due to gravity. With the dynamic boundary condition $p=0$, and the kinematic boundary condition $\partial_{t} \eta+\left(\partial_{X_{1}} \eta\right) v_{1}+\left(\partial_{X_{2}} \eta\right) v_{2}=v_{3}$, we obtain the following system of nonlinear boundary conditions at the free surface:

$$
\varphi_{t}+\frac{1}{2}|\nabla \varphi|^{2}+g \eta=0, \quad \eta_{t}+\nabla_{X} \eta \cdot \nabla_{X} \varphi=\varphi_{Y}, \quad \text { at } \Gamma_{s} .
$$

The remaining boundary conditions are given by the velocity of the wavemaker, an impermeability condition at all fixed boundaries, and a radiation condition 
for the open domain,

$$
\begin{aligned}
& \frac{\partial \varphi}{\partial n}=f(t)= \begin{cases}-a^{\prime}(t) & \text { at } \Gamma_{w}, \\
0 & \text { at } \Gamma_{0}, \Gamma_{1},\end{cases} \\
& \frac{p}{\varrho}+g Y=0 \Leftrightarrow \varphi_{t}+\frac{1}{2}|\nabla \varphi|^{2}=0 \text { at } \infty .
\end{aligned}
$$

\section{Transformation of the mathematical problem}

In this section, we derive a nonlocal system of partial differential equations for the velocity potential and the free surface. We denote by $W$ the projection of the potential to the free surface, $W(X, t)=\varphi(X, \eta(X, t), t)$. From (2) we obtain

(4) $\quad W_{t}=-\frac{1}{2}|\nabla W|^{2}+\frac{1}{2} \varphi_{Y}^{2}\left(1+|\nabla \eta|^{2}\right)-g \eta$,

$$
a(t)<X_{1}<K(t), 0<X_{2}<b, t>0,
$$

(5) $\eta_{t}=-\nabla W \cdot \nabla \eta+\varphi_{Y}\left(1+|\nabla \eta|^{2}\right)$,

$$
a(t)<X_{1}<K(t), 0<X_{2}<b, t>0,
$$

with the initial condition

$$
W(X, 0)=0, \quad \eta(X, 0)=0,
$$

where $\nabla=\left(\partial / \partial X_{i}\right)_{i}$, and $K(t)$ is the $X_{1}$-coordinate of the contact point between $\Gamma_{s}$ and $\Gamma_{1}$ in the two-dimensional case (see Fig. 2). Obviously, $K(t)$ is constant 
for a vertical wall $\Gamma_{1}$. These equations are augmented by the continuity equation $\Delta \varphi=0$ in $\Omega(\eta, a)$, with the boundary conditions

$$
\frac{\partial \varphi}{\partial n}=\left\{\begin{array}{ll}
-a^{\prime}(t) & \text { at } \Gamma_{w}, \\
0 & \text { at } \Gamma_{0}, \Gamma_{1},
\end{array} \text { and } \varphi=W \quad \text { at } \Gamma_{s} .\right.
$$

A similar formulation was used, e.g., in [11] in a Hamiltonian approach to the classical water wave problem.

With the coordinate transformation $\Phi: \Omega(s) \rightarrow \Omega(\eta, a)$,

$$
\begin{gathered}
X_{1}=a(t)+\frac{K(t)-a(t)}{K} x_{1}, \quad Q(t)=\frac{\partial(x, y)}{\partial(X, Y)}=\left(\begin{array}{cc}
Q^{x}(t) & 0 \\
0 & 1
\end{array}\right), \\
Q^{x}(t)= \begin{cases}\left(\begin{array}{cc}
q(t) & 0 \\
0 & 1
\end{array}\right) & \text { for } x \in \mathbb{R}^{3}, \quad q(t)=\frac{K(0)}{K(t, s)-a(t)}, \\
q(t) & \text { for } x \in \mathbb{R}^{2},\end{cases}
\end{gathered}
$$

we are able to describe system (4), (5) in a fixed domain (see Fig. 2). Introducing the functions

$$
s(x, t):=\eta(X, t), \quad w(x, t):=W(X, t), \quad u(x, y, t):=\varphi(X, Y, t),
$$

we obtain a system of nonlocal, partial differential equations of first order for $w$ and $s$ in $B=(0, K(0)) \times(0, b) \times \mathbb{R}_{+}$:

$$
\begin{aligned}
s_{t}= & u_{y}-\left(Q^{x} \nabla s \cdot Q^{x} \nabla w\right)+u_{y}^{2}\left|Q^{x} \nabla s\right|^{2}+s_{x_{1}} \widetilde{q} \\
w_{t}= & u_{y} s_{t}-\left(w_{x_{1}}-u_{y} s_{x_{1}}\right) \widetilde{q}+\frac{1}{2}\left(u_{y}^{2}-\left|Q^{x} \nabla w\right|^{2} u_{y}^{2}\left|Q^{x} \nabla s\right|^{2}\right) \\
& +u_{y}\left(Q^{x} \nabla s \cdot Q^{x} \nabla w\right)-g s
\end{aligned}
$$

with $\widetilde{q}=q(t) a^{\prime}(t) \frac{x-K(0)}{K(0)}$ and the initial condition

$$
s(x, 0)=0, \quad w(x, 0)=0, \quad x \in B .
$$

The nonlocal term, $u_{y}$, is uniquely determined by the solution $u$ to

$$
\nabla\left(Q^{2} \nabla u\right)=0 \quad \text { in } \Omega(s),
$$

with the boundary conditions

$$
\begin{array}{ll}
n \cdot Q^{2} \nabla u=f(t) & \text { at } \Gamma_{0}, \Gamma_{1}, \Gamma_{w}, \\
u=w & \text { at } \Gamma_{s} .
\end{array}
$$

The above described reduction of system (1) splits the problem into a linear and a nonlinear part. The linear operator, given by the equations (9)(10), is formulated in the time dependent domain $\{(x, y, t) \mid(x, y) \in \Omega(s)\} \subset$ $\mathbb{R}^{r} \times \mathbb{R}_{+}$, whereas the nonlinear operator, given by the equations (6)-(8), describes the velocity potential and the free surface in the fixed domain $\{(x, t) \mid$ $x_{1} \in(0, K(0)\} \subset \mathbb{R}^{r-1} \times \mathbb{R}_{+}, r=1,2$. Note that the regularity of the solution 
is determined by the partial differential equations in (1). The transformation of the problem does not require any higher regularity.

\section{Numerical solution}

The system (6)-(9) is a highly nonlinear system of partial differential equations. The stability and convergence of a numerical scheme for its approximation intrinsicly depends on the choice of the discretization for the right hand side of the system. We developed two numerical programs, one for the solution of the conservation law in a time dependent domain and the second for the solution of the equations of motion and the free surface condition. Both programs were tested independently on several theoretical problems with known exact solutions. Based on these tests we derived a method for the solution of the coupled problem which was successfully used for long-time calculations of free surface waves.

We apply the method of lines to (6)-(10) to derive a system of ordinary differential equations for the vector

$$
Z_{i}(t)=S_{i}(t)=s\left(x^{i}, t\right), \quad Z_{i+n x}(t)=W_{i}(t)=w\left(x^{i}, t\right), \quad i=0, \ldots, n x-1 .
$$

We denote by $U_{i}(t)$ the restriction of the velocity potential to the grid points on the free surface.

The vector $\left(x^{i}\right)_{i=0, \ldots, n x-1}$ contains the grid points of an equidistant, horizontal mesh, where $n x$ denotes the number of grid points. For the discretization of $\nabla s$ and $\nabla w$ we use modified upwind schemes. To discretize $s_{x}^{2}$, we apply the relation $s_{x}^{2}=\left(s_{x} s\right)_{x}-s_{x x} s$. After the space discretization we obtain the following system of ordinary differential equations in the two-dimensional case:

$$
\begin{aligned}
Z_{i}^{\prime}(t)= & U_{i y}(t) \\
& +\left\{d_{i}\left(\nabla^{+} S_{i}\right)(t)+\left(1-d_{i}\right)\left(\nabla^{-} S_{i}\right)(t)\right\} \\
& \times\left\{\frac{a^{\prime}(t)\left(x^{i}-K(0)\right) q(t)}{K(0)}-\left(\nabla^{ \pm} W_{i}\right)(t) q(t)^{2}\right\} \\
& +2 U_{i_{y}}(t)\left\{\nabla^{-}\left[\left(\nabla^{+} S_{i}\right)(t) \bar{S}_{i}(t)\right]-\bar{S}_{i}(t)\left[\nabla^{-}\left(\nabla^{+} S_{i}\right)(t)\right]\right\},
\end{aligned}
$$

(12) $\quad Z_{i+n x}^{\prime}(t)=U_{i y}(t) Z_{i}^{\prime}(t)$

$$
\begin{aligned}
& -\frac{\left\{\left(\nabla^{ \pm} W_{i}\right)(t)-U_{i y}(t)\left(\nabla^{ \pm} S_{i}\right)(t)\right\} a^{\prime}(t)\left(x^{i}-K(0)\right) q(t)}{K(0)} \\
& -\frac{\left\{\left(\nabla^{ \pm} W_{i}\right)(t)-U_{i y}(t)\left(\nabla^{ \pm} S_{i}\right)(t)\right\}^{2} q(t)^{2}+U_{i y}(t)^{2}}{2}-g S_{i}(t),
\end{aligned}
$$

where

$$
d_{i}=H\left(-\left(\nabla^{ \pm} W_{i}\right)(t) q(t)^{2}+\frac{a^{\prime}(t)\left(x^{i}-K(0)\right) q(t)}{K(0)}\right), \quad \bar{S}_{i}=\frac{1}{2}\left(S_{i}+S_{i+1}\right),
$$

$H$ is the Heaviside function and $\nabla^{+}, \nabla^{-}, \nabla^{ \pm}$denote forward, backward and central finite differences, respectively. The resulting system of ordinary differential 
Figure 3. The wave formation near the wavemaker 
equations for $Z(t)$ can be solved with a highly efficient method. We implemented backward differentiation formulas from the package LSODE [8]. Note that the Jacobian for the right hand side of system (11)-(12) is a full matrix due to the global effect of the velocity potential $U_{i}$. To avoid time expensive approximations of this matrix, we use fixed point methods for the solution of the underlying nonlinear systems of equations. To calculate the right hand side of (11)-(12) we have to supply the derivatives of the velocity potential in vertical direction, $U_{i y}$, at all grid points $x_{i}$. At any time level, this potential is uniquely determined by $W, S$ and the boundary conditions (10) as a solution of the continuity equation (9). We solve the continuity equation using a finite volume method on an adaptive time dependent grid. This adaptive grid is automatically generated dependent on the discrete solution of the function $s$ (see Fig. 9).

We have chosen an implicit numerical method to obtain a possibly accurate approximation for the nonlinear system of equations and to satisfy natural compatibility conditions at the interfaces and boundaries. In the following sections, we present a series of numerical simulations of two-dimensional water waves under different boundary conditions.

The motion of water and the formation of free surface waves are caused by the enforced motion of the wavemaker. The interaction between the boundary velocity, the velocity potential and the free surface at the beginning of the process are presented in Figs. 3-4.

\section{Reflecting boundary conditions}

In the first set of calculations we simulate the motion of water in a small wave tank with reflection at the boundaries. At the beginning the water is still and the free boundary is a plane surface. The periodic motion of the wavemaker causes a continuous formation of waves. These waves are reflected at the opposite boundary and interact nonlinearly with the incoming waves (Figs. 5-6).

In the next calculations (Figs. 8-7) the wavemaker stopped after one period of motion. The initiated high wave is followed by some smaller reflection waves. As the time proceeds, the process is characterized by the interaction of waves of different amplitudes and velocities.

\section{Absorbing boundary conditions}

The above described method can be coupled in a natural way with an absorbing boundary condition at an artificial boundary to simulate the solution in an open domain. The radiation condition in (3) can be derived from the assumption that there is no energy source at infinity. The formal transformation of this condition to a finite domain forces the free surface to the still water level 
FIGURE 5. Free surface waves caused by a periodic motion of the wavemaker and reflection

Figure 6. 2D view of Fig. 5 
A. Pawell - R. B. Guenther

FiguRE 7. Free surface waves cased by a short motion of the wavemaker and reflection

Figure 8. 2D view of Fig. 7 
and causes reflection. The energy condition does not determine boundary conditions at any artificial boundary inside the domain of fluid motion. For linearized waves, boundary conditions can be derived which filter out the incoming waves and simulate an undisturbed outflow. Similar conditions have been successfully applied to nonlinear wave systems. For an overview concerning absorbing boundary conditions we refer to [3], [7], [10], [14].

The implementation of the above described splitting method requires a boundary condition for the continuity equation at an artificial boundary $\Gamma_{1}=$ $\left\{(x, x) \mid x_{1}=K(0)\right\}$. This boundary condition has a global effect on the solution of the system. To minimize the influence of the boundary $\Gamma_{1}$, we extend the calculation domain $\Omega(s)$ in $x_{1}$-direction and solve the continuity equation in the extended domain $\Omega(s) \cup \widetilde{\Omega}(\widetilde{s})$. Without loss of accuracy, this can be done with a coarser grid in $\Omega(\widetilde{s})$. For the presented calculations we have chosen a grained grid with raising step-size outside $\Omega(s)$ (see Fig. 9).

$\begin{array}{ccc}\text { Calculation } & \text { Artificial } & \\ \text { domain } & \text { boundary } & \text { Outer } \\ \Omega(s) & x_{1}=K(0) & \text { domain } \\ \widetilde{\Omega}(\widetilde{s})\end{array}$

FiguRE 9. Extension of the calculation domain

We solve the nonlinear system of equations of motion and the free surface condition only in $\Omega(s)$. To provide $\widetilde{s}$ and boundary conditions for the continuity equation in $\Omega(\widetilde{s})$ we use a traveling wave assumption to extend the free surface and the velocity potential at the boundary to the range $x_{1}>K(0)$.

Supposing $\eta(x, t)=E(x-c t)$, we calculate the velocity of outgoing waves from $c \eta_{x}=\eta_{t}$ in the neighborhood of the artificial boundary. Then the boundary conditions for $x_{1}>K(0)$ are given by

$$
\eta(\tilde{X}, \tilde{t})=\eta(\widetilde{X}-c(\widetilde{t}-t), t) \quad \text { and } \quad W(\widetilde{X}, \widetilde{t})=W(\widetilde{X}-c(\widetilde{t}-t), t)
$$

Figs. 10-13 represent calculations of water waves with absorbing boundary conditions at $\Gamma_{1}$. In Figs. 10-11 the waves are caused by a periodic motion of the wavemaker.

The waves in Figs. 12-13 are caused by the short motion of the wavemaker which stops after one period at the rest position. The initiated wave and following small reflection waves are traveling out of the domain of calculation. 
A. Pawell - R. B. Guenther

Figure 10. Free surface waves caused by a periodic motion of the wavemaker and absorption

Figure 11. 2D view of Fig. 10 
Figure 12. Wave propagation in an open domain

Figure 13. 2D view of Fig. 12 


\section{Comparison with experiment ${ }^{1}$}

A detailed discussion of experimental work would be out of place here and will be published elsewhere. However, we shall give the results of one simple experiment which can be used to validate the model. A two-dimensional crosssection of the wave tank has the form as sketched in Fig. 14.

FigurE 14. Wave tank near wavemaker (not to scale)

Figure 15. Top view of the wave tank near wavemaker (not to scale)

Small waves were generated using a hinged or flap wavemaker. The wavemaker began its cyclic motion when the tank had been filled to a depth of 3.5052 $\mathrm{m}$ and allowed to come to rest. The amplitude (or semi-stroke) of the wavemaker at the still water level was $.025014 \mathrm{~m}$. The frequency $f$, which is one divided by the period, was $1.297 \mathrm{~Hz}$ so that the circular frequency $\omega=2 \pi f$ was $8.1493 \mathrm{~s}^{-1}$. Wave gauges were placed at various locations in the wave tank. These measured the wave heights at that location as a function of time. The location of the wave gauge used here is shown in Fig. 15.

The time series given in Fig. 6 show the wave heights measured by the wave gauge, where the zero on the height scale has been taken to be the still water level. The results of a complete simulation are pictured in Figs. 17-19.

\footnotetext{
${ }^{1}$ We wish to thank D. Stanley and T. Dibble of the Oregon State University, ONR-Hinsdale Wave Facility for making this data available to us.
} 
FigURE 16. Wave heights measured by the wave gauge

Figure 17. Wave heights simulated using data corresponding to Fig. 16 
Figure 18. Time and length dependent plot of wave heights $2-20 \mathrm{~m}$ away from the wave board 


\section{REFERENCES}

[1] J. T. BEALE, The initial value problem for the Navier-Stokes equations with a free surface, Comm. Pure Appl. Math. 34 (1981), 359-392.

[2] Large-time regularity of viscous surface waves, Arch. Rational Mech. Anal. 84 (1984), 307-352.

[3] J. Broeze And J. E. Romate, Absorbing boundary conditions for free surface waves, Simulations with a panel method, J. Comput. Phys. 99 (1992), 146-158.

[4] M. Dauge, Elliptic Boundary Value Problems on Corner Domains, Lecture Notes in Math., vol. 1341, Springer-Verlag, Berlin, 1988.

[5] P. Grisvard, Elliptic Problems in Nonsmooth Domains, Pitman Adv. Publ. Program, 1985.

[6] Singularities in Boundary Value Problems, Res. Notes Appl. Math., vol. 22, Springer-Verlag, 1992.

[7] R. L. Higdon, Radiation boundary conditions for dispersive waves, SIAM J. Numer. Anal. 31 (1994), 64-100.

[8] A. C. Hindmarsh, Lsode and lsodi, two new initial value ordinary differential equation solvers, ACM Newsletter 15 (1980), 10-11.

[9] V. A. Kondrat'ev And O. A. OLEǏnik, Boundary value problems for partial differential equations in nonsmooth domains, Uspekhi Mat. Nauk 38 (1983), no. 2, 1-86. (Russian)

[10] D. Kröner, Absorbing boundary conditions for the linearized Euler equations in 2-d, Math. Comput. 57 (1991), 153-167.

[11] A. C. RADDER, An explicit Hamiltonian formulation of surface waves in water of infinite depth, J. Fluid Mech. 237 (1992), 435-455.

[12] J. REEDER And M. Shinbrot, The initial value problem for surface waves under gravity, II The simplest 3-dimensional case, Indiana Univ. Math. J. 25 (1976), 1049-1071.

[13] The initial value problem for surface waves under gravity, III Uniformly analytic initial domains, J. Math. Anal. Appl. 67 (1979), 340-391.

[14] J. E. Romate, Absorbing boundary conditions for free surface waves, J. Comput. Phys. 99 (1992), 135-145.

[15] M. Shinbrot, The initial value problem for surface waves under gravity, I. The simplest case, Indiana Univ. Math. J. 25 (1976), 281-300.

[16] J. Socolowsky, Solvability of a stationary problem on the plane motion of two viscous incompressible liquids with non-compact free boundaries, Z. Angew. Math. Mech. 72 (1992), 251-268.

[17] Existence and uniqueness of the solution to a free boundary-value problem with thermocapillary convection in an unbounded domain, Acta Appl. Math. 37 (1994), 181194.

[18] The solvability of a free boundary problem for the stationary Navier-Stokes equations with a dynamic contact line, Nonlinear Anal. 21 (1993), 763-784.

[19] V. A. Solonnikov, Solvability of a problem on the motion of a viscous incompressible fluid bounded by a free surface, Math. USSR-Izv. 11 (1977), 1323-1358. 
[20] A. TANi, Free boundary problems for the incompressible Euler equations, Surrikaisekikenkyûsho Kôkyûroku 862 (1994), 237-248.

Manuscript received January 4, 1996

A. PAWEll

Institut für Mathematik

Technische Universität Cottbus

PF 101344, 03013 Cottbus, GERMANY

E-mail address: pawell@math.tu-cottbus.de

R. B. Guenther

Department of Mathematics

Oregon State University

Corvallis, OR 97331, USA

E-mail address: guenth@math.orst.edu 